\title{
Quantitative regional curvature analysis: A prospective evaluation of ventricular shape and wall motion measurements
}

\author{
G. B. John Mancini, MD, Scott F. DeBoe, BS, Mark J. McGillem, BS, and \\ Eric R. Bates, MD. Ann Arbor, Mich.
}

Wall motion analysis is a fundamental prerequisite for both clinical decision-making and for evaluating the results of new therapy or interventions. Traditional methods used to measure wall motion per se can all be characterized by the need to invoke one or more assumptions about idealized geometry of the ventricle, the reference system (internal or external), the indexing system (fixed or floating), and the coordinate system (radial or perpendicular). ${ }^{1}$ Consequently, numerous approaches have been proposed that are all dramatically affected by translational and rotational motion within the image plane. Our laboratory has attempted to circumvent these problems by measuring ventricular shape as an indirect index of actual function. The approach is based on the hypothesis that normal and abnormal ventricles look dissimilar by virtue of differences in regional function and that clinicians use these shape cues when assessing ventricular function. Therefore a measure of shape should provide an index related to ventricular function. Our laboratory has previously established normal shape patterns, ${ }^{2}$ patterns of abnormality, ${ }^{3}$ and the performance of the shape algorithm compared to that of the centerline method. ${ }^{4}$ The purpose of this investigation was to reevaluate prospectively the comparative performance of these two approaches for the diagnosis of subtle abnormalities of ventricular function in a population different from the population used for initial development of the algorithms. The study also

From the Department of Internal Medicinc, Division of Cardiology, Veterans Administration Medical Center, University of Michigan Medical School.

This study was supported in part bv funds from the Veterans Administration, Washington D.C., the American Heart Association of Michigan, Lathrup Village, Michigan, and by NIH1ROIHL36813, Bethesda, Md. Received for publication May 13, 1988; accepted July 19, 1988.

Reprint requests: G. B. John Mancini, MD, Veterans Administration Medical Center (111A), 2215 Fuller Road, Ann Arbor, MI 48105. compares the degree to which each method simulates the performance of clinical observers and the prerequisites for achieving this level of performance. The ultimate goal of this line of work is to begin to develop methods for automatic analysis of ventricular function beginning with the stage of edge detection and proceeding through to the stage of generating a probabilistic interpretation within the context of utilizing artificial intelligence techniques for decision-making.

\section{METHODOLOGY}

The study group consisted of 82 patients, of which 30 had been used to establish a normal data base ${ }^{4}$ and the remaining 52 were new patients with normal or mildly abnormal regional ventricular function. Patients with akinesis, dyskinesis, or hyperkinesis were not included. End-diastolic and end-systolic outlines of the 52 new patients were drawn with an external reference system convention to allow application of the centerline method. ${ }^{5}$ These were viewed by four independent observers on two occasions. The original ventriculograms were not shown in order to present only the static outlines, thereby mandating that all clinical decisions would depend solely on shape and displacement cues and not on cues related to the coordination of motion. Two observers were familiar with the quantitative regional curvature algorithm (GBJM, SFD) and two were not (MJM, ERB). Each observer was asked to classify the anterior, apical, and inferior regions as either normal ( 0 points) or abnormal ( 1 point). Scores were collated so each segment was assigned a semiquantitative visual score ranging from 0 (all four nhservers felt that the segment was normal on both readings) to 8 (all four observers felt that the segment was abnormal on both readings). There were seven instances of apical abnormalities and these were invariably associated with anterior 

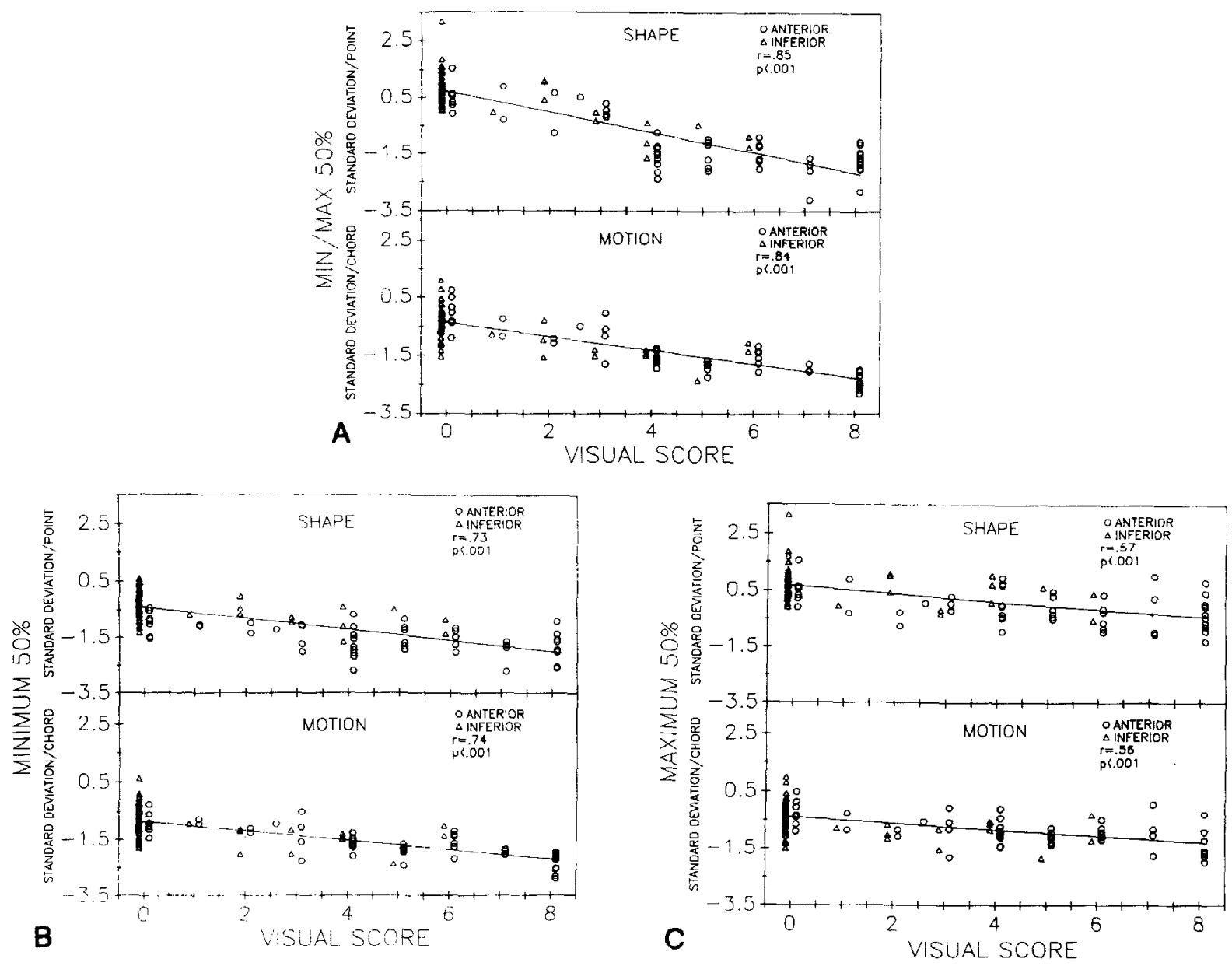

Fig. 1. Panels $\mathbf{A}, \mathbf{B}$, and $\mathbf{C}$ show the results of the different quantitative applications for both the shape and wall motion algorithms. Optimal results were obtained with the " $\mathrm{min} / \mathrm{max}$ " approach (panel A). Shape and motion quantitation were equal in their relation to the visual scores.

abnormalities. Therefore the scores for these two segments were added and then halved and the segments were considered to be anterior segments for the remainder of the study. This method provided a continuous variable for assessing the degree of abnormality. For categorical analyses, a visual score of $\geq 4$ was used to designate an abnormal segment. This grading resulted in 62 normal segments (16 anterior and 46 inferior) and 42 abnormal segments (36 anterior and 6 inferior). No attempt was made to establish a cohort with an equal distribution of normal and abnormal regions.

The outlines were then quantitated with the use of the previously detailed centerline method $^{5,6}$ and the quantitative regional curvature analysis method. ${ }^{2-4}$ Based on prior experience, only the endsystolic shape was examined with the quantitative regional curvature analysis method. $0^{3,4}$ Quantitation of motion and shape was constrained to $50 \%$ of the segment length, as previously described. ${ }^{4-6}$ Each algorithm was applied in three different ways: (1) the minimum ("worst") value within $50 \%$ of the segment length; (2) the maximum ("best") value within $50 \%$ of the segment length; and (3) based on the clinical designation, the minimum value was quantitated when the segment was felt to be clinically abnormal and the maximum value was quantitated when the segment was felt to be clinically normal., ${ }^{4,5}$ This was called the "min/max" approach. Motion results were reported in units of standard deviations per chord and shape results were reported in units of standard deviations per point.

Each quantitative methodology was analyzed to determine the critical value that maximized performance based on sensitivity, specificity, and concordance. In the absence of a true "gold standard" for 

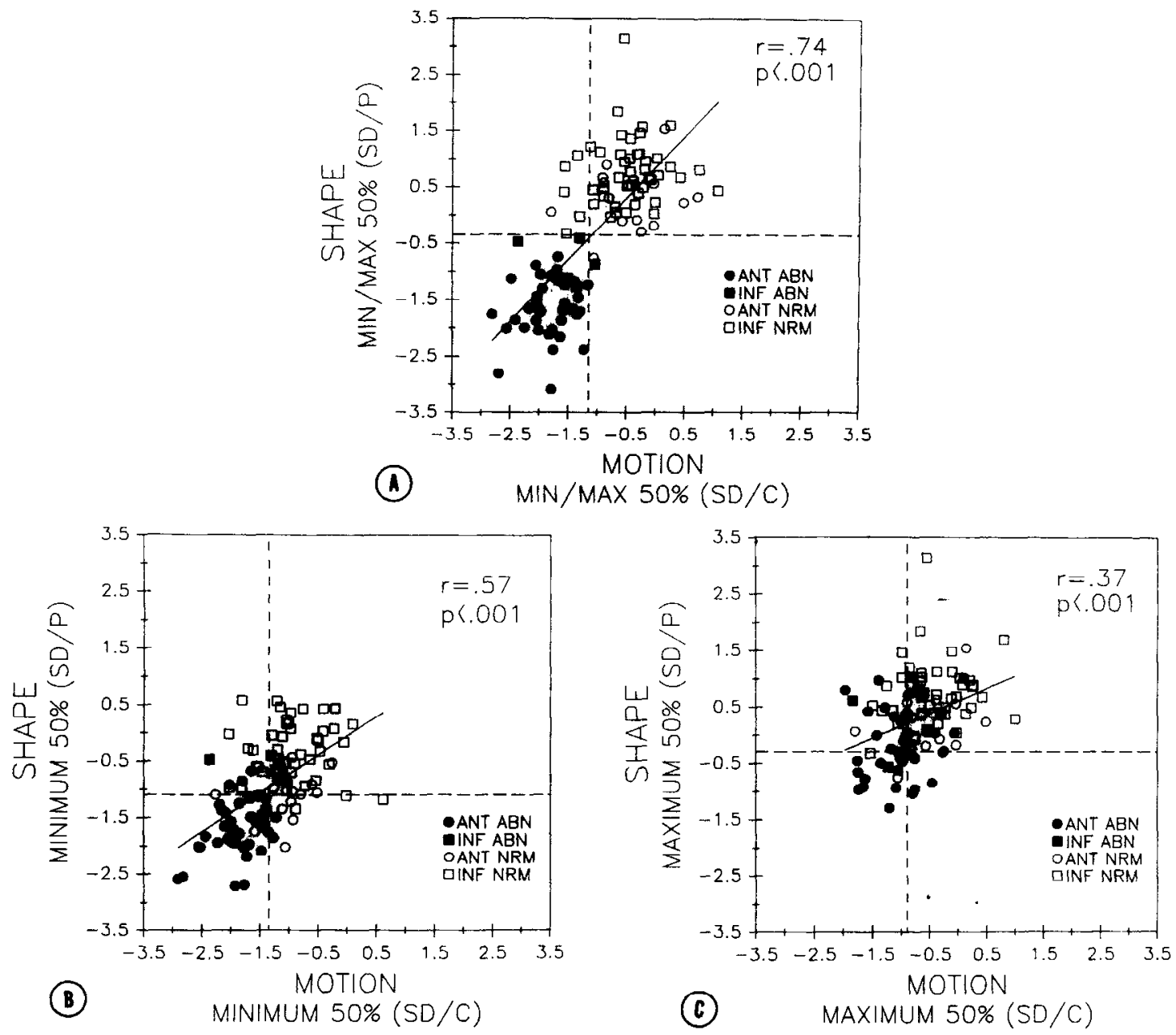

Fig. 2. Panels $\mathbf{A}, \mathbf{B}$, and $\mathbf{C}$ show the relation between motion and shape scores. Optimal results were obtained with the "min/max" approach (panel A). Dashed lines show the critical values determined for each technique (see Table I). $A N T$, anterior; $I N F$, inferior; $N R M$, normal; $A B N$, abnormal.

regional function, the clinical designation was used as a "gold standard." This was further justified because one of the intents was to develop a system that mimics clinical performance. Accordingly, sensitivity was defined as the number of quantitatively abnormal segments divided by the total number of clinically abnormal segments. Specificity was defined as the number of quantitatively normal segments divided by the total number of clinically normal segments. Concordance was defined as the number of segments that were both clinically and quantitatively normal or abnormal divided by the total number of segments.

Linear regression analysis was used to determine the relation between visual scores and both shape and wall motion scores. This was also used to determine the relation between shape and motion scores. Categorical analyses for sensitivity, specifici- ty, and concordance rates were analyzed with a chi square test. $p$ values $<0.05$ were considered significant.

\section{OBSERVATIONS}

Fig. 1 shows the regression results between shape scores or wall motion scores and visual scores. Optimal correlations were obtained by means of the "min/max" approach. Both the shape and motion results correlated to a similar degree with the clinical designation, irrespective of the quantitative approach used.

Fig. 2 showe the relation between motion and shape quantification. As in the analyses shown in Fig. 1, results of this analysis were also best when the "min/max" approach was used. Notice in panel $A$ that one inferior segment was shown to have abnormal shape but normal wall motion. The 
Table I. Critical values, sensitivity, specificily, and concordance rates for each application of the shape and wall motion algorithms

\begin{tabular}{lcccc}
\hline & $\begin{array}{c}\text { Critical } \\
\text { value }\end{array}$ & Sensitivity & Specificity & $\begin{array}{c}\text { Con- } \\
\text { cordance }\end{array}$ \\
\hline Approach & & & & \\
"Min/max" 50\% & & & & \\
$\quad$ Shape & -0.35 & 100 & 98.4 & 99.0 \\
$\quad$ Motion & -1.15 & 97.6 & 90.3 & $93.3 \dagger$ \\
Minimum" 50\% & & & & \\
$\quad$ Shape & -1.10 & 85.7 & 83.9 & 84.6 \\
$\quad$ Motion & -1.35 & 90.5 & 80.7 & 84.6 \\
"Maximum" 50\% & & & & \\
$\quad$ Shape & -0.30 & 45.2 & 96.8 & 76.0 \\
$\quad$ Motion & -0.90 & 64.3 & $82.3^{*}$ & 75.0 \\
\hline
\end{tabular}

Critical values are given in units of standard deviations per point (shape) and units of standard deviations per chord (motion).

Results are given as percentages. See text for definition of terms.

${ }^{*} p<0.01$ versus shape analysis.

$\dagger p<0.04$ versus shape analysis.

quantitative output for this patient is shown in Fig. 3.

Table I shows the critical values, sensitivity, specificity, and concordance results for all three approaches. The "min/max" approach was again optimal for both techniques. In this instance, concordance was significantly better for the shape approach $(99 \%$ versus $93 \%, p<0.04)$. Sensitivity was equal ( $100 \%$ vs $98 \%, p=0.31)$ and specificity was not quite significantly different $(98 \%$ vs $90 \%$, $p=0.052$ ).

\section{COMMENTS}

This laboratory has attempted to take a radically different approach to solving the clinical problem of regional function quantitation in order to circumvent the assumptions and approximations required by traditional wall motion methodologies. The method of using shape analysis is based on the hypothesis that clinicians use shape cues to assess regional function and that normal and abnormal ventricles look different by virtue of differences in function. Accordingly, methods incorporating shape analysis should provide methodologies that mimic clinical performance and that provide quantitative parameters that reflect regional ventricular function.

This study evaluates the comparative performance of the quantitative regional curvature analysis method and the centerline method in a new population consisting primarily of patients with normal or minimally abnormal regional function. The results demonstrate that: (1) both approaches have similar overall performance but the shape
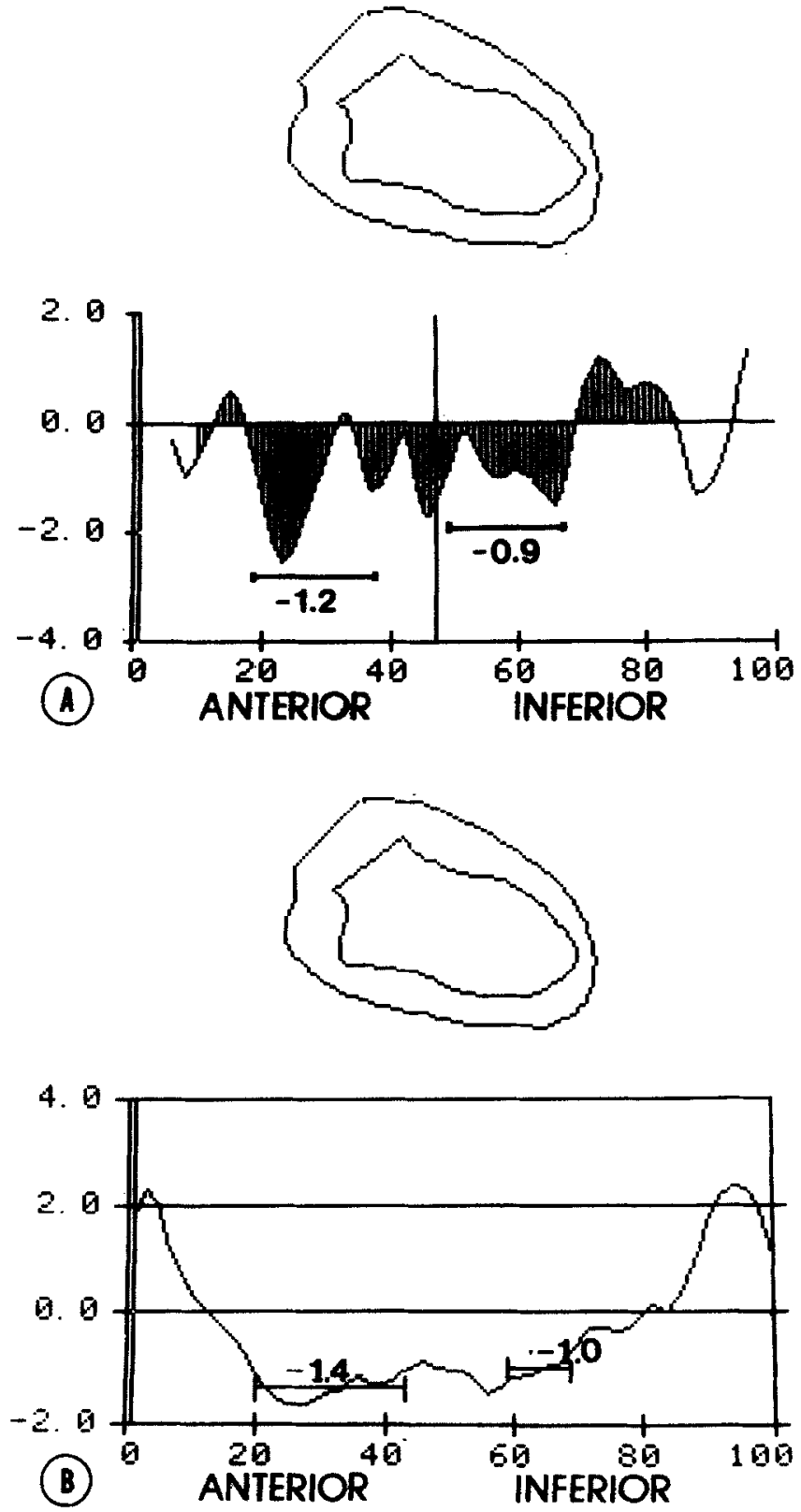

Fig. 3. Shape and motion analyses for an individual are shown. The $\mathrm{x}$ axis shows the location of the abnormality and the $y$ axis shows the deviation from normal in units of standard deviation (panel $A=$ shape analysis, panel $B=$ motion analysis). This patient was clinically and quantitatively found to have mildly abnormal function of the anterior wall. The clinically apparent, mild inferior abnormality was detected only by shape analysis (panel A).

analysis method mimics clinical performance somewhat better when both methods are applied to maximize the likelihood of finding normal and abnormal results (i.e., the " $\mathrm{min} / \mathrm{max}$ " approach) ${ }^{4-6}$; (2) both motion and shape quantitation showed similar linear relations to the probabilistic, visual 
quantitation of regional function; and (3) although the shape and wall motion scores correlate with each other, there are instances when sole reliance on wall motion analysis caused disagreements with the clinical interpretation.

The results of the current study confirm previously published results ${ }^{4}$ obtained in a different study population. Considering the results of the "min/ max" approach previously obtained, the correlation between shape and motion parameters was 0.748 compared to 0.74 in the current study, the critical value for shape analysis was -0.4 standard deviations per point compared to -0.35 in the current study, and the critical value for motion analysis was -1.10 standard deviations per chord compared to -1.15 in the current study. Similar concordance rates were also previously found for shape analysis (96.5\% compared to $99.0 \%$ in this study) and motion analysis (93.9\% compared to $93.3 \%$ ). In summary, consistent performance of both methodologies was demonstrated.

Aside from providing confirmatory results, this investigation has several other important implications. First, the traditional approaches have led to continuing controversies regarding the relative merits of fixed and internal reference frames, coordinate systems, and indexing systems but without a resultant general consensus., ${ }^{1,8}$ All of the traditional methods are markedly affected by translational and rotational motion parallel to the imaging plane. The shape analysis methodology is the first quantitative approach that is not affected by these factors, thereby obviating long-standing reservations about the reliability and accuracy of traditional wall motion methods. Second, since the quantitative regional curvature analysis can be applied throughout the cardiac cycle ${ }^{9}$ and because the results are independent of size, ${ }^{2-4}$ the opportunity exists to assess both systolic and diastolic function independent of volume. Third, the visual cues used by clinicians to make regional function interpretations can be quantitated and therefore can be incorporated into robust methods for achieving automatic edge detection and decision-making. Fourth, the ability to measure shape provides a tool for serial assessment of remodelling of the ventricle after myocardial infarction and to assess potential alteration of this process by drugs and therapeutic interventions. ${ }^{10}$ Finally, shape analysis, ${ }^{10,11}$ perhaps through its relation to regional wall stress, ${ }^{12}$ may be more important prognosticaily than simple measures of global or regional function.

One of the great limitations of both methods is that optimal results are achieved only when a priori knowledge is used to determine the specific way in which the programs are to be applied. For example, the centerline method was validated by quantitating the area of worst abnormality in zones supplied by abnormal coronary arteries and by quantitating the area of least abnormality in zones supplied by normal coronary arteries. ${ }^{5.6}$ The quantitative regional curvature analysis also shows optimal performance when applied in a similar fashion. ${ }^{4}$ Further work is necessary to free these methods of this requirement. One approach might be to generate automatically the probability of whether a segment is normal or abnormal based on its shape and to proceed with wall motion analysis based on this information alone. This approach would be analogous to template matching and might become a key procedure for automatic wall motion interpretation. $^{13}$

\section{SUMMARY}

To overcome the assumptions and approximations mandated by the use of traditional wall motion methodologies, a method was recently developed for measuring ventricular shape based on quantitative curvature analysis of ventricular outlines. This study was designed to assess prospectively the performance of this algorithm, to compare it to traditional wall motion measurements (centerline method), and to determine the comparative degree to which each method mimicked the interpretation of wall motion by clinical observers. Semiquantitative visual grading of regional function in 52 patients was performed by four independent observers on two occasions. Anterior, apical, or inferior segments were judged to be normal ( 0 points) or abnormal ( 1 point) based on viewing nonrealigned, end-diastolic and end-systolic ventricular silhouettes from cineventriculograms obtained in the 30-degree right anterior oblique projection. Each segment was assigned a collated score ranging from 0 (all observers felt the region was normal on both readings) to 8 (all observers felt the region was abnormal on both readings). Quantitative regional curvature analysis and wall motion analysis (centerline method) were performed. Quantitative shape and wall motion scores correlated equally well with the semiquantitative visual scores. When a visual score of $\geq 4$ was used to designate an abnormal segment, both quantitative approaches demonstrated comparable sensitivity, specificity, and concordance rates. Both methods achieved optimal performance when maximum and minimum deviations from normal were recorded. Under these circumstances, the shape analysis demonstrated a greater concordance with 
the clinical diagnosis than did wall motion analysis (99\% vs $93 \%, p<0.04)$. Thus new information is provided by the shape analysis program that reflects clinical evaluations more closely and does not require assumptions mandated by traditional wall motion methods. This confirms the value of quantitative regional curvature analysis in a prospectively studied patient population with subtle wall motion abnormalities.

\section{REFERENCES}

1. Ingels NB, Daughters GT, Stinson EB, Alderman EL. Evaluation of methods for quantitating left ventricular segmental wall motion in man using myocardial markers as a standard. Circulation 1980;61:966.

2. Mancini GBJ, LeFree MT, Vogel RA. Curvature analysis of normal ventriculograms: fundamental framework for the assessment of shape changes in man. Comput Cardiol 1985; 141.

3. Mancini GBJ, DeBoe SF, Anselmo E, Simon SB, LeFree MT Vogel RA. Quantitative regional curvature analysis: an application of shape determination for the assessment of segmental function in man. AM Heart J 1987;113:326.

4. Mancini GBJ, DeBoe SF, Anselmo E, LeFree MT. A comparison of traditional wall motion assessment and quantitative shape analysis: a new method for characterizing left ventricular function in humans. AM HEART J 1987;114:1183.
5. Sheehan FH, Dodge HT, Mathey DG, Bolson EL, Mitten S. Application of the centerline method: analysis of change in regional left ventricular wall motion in serial studies. Comput Cardiol 1980; :97.

6. Bolson EL, Kilman S, Sheehan FH, Dodge HT. Left ventricular segmental wall motion: a new method using local direction information. Comput Cardiol 1980;245.

7. Karsch KR, Lamm U, Blanke H, Rentrop KP. Comparison of nineteen quantitative methods for assessment of localized left ventricular wall motion abnormalities. Clin Cardiol 1980;3:123.

8. Gelberg JH, Brundage BH, Glantz S, Parmley WW. Quantitative left ventricular wall motion analysis: comparison of area, chord and radial methods. Circulation 1979;59:991.

9. Mancini GBJ, Simon SB, DeBoe SF, LeFree MT. Quantitative regional curvature analysis: assessment of regional shape abnormalities throughout systole and diastole [Abstract]. Clin Res 1987;35:877A.

10. Weisman HF, Healy B. Myocardial infarct expansion, infarct extension, and reinfarction: pathophysiologic concepts. Prog Cardiovasc Dis 1987;30:73.

11. Jugdutt BI. Can the degree of regional shape distortion on an early two-dimensional echocardiogram after myocardial infarction identify patients prone to infarct expansion? [Abstr]. J Am Coll Cardiol 1986;7:149A.

12. Gould KL, Lipscomb K, Hamilton GW, Kennedy JW. Relation of left ventricular shape, function and wall stress in man. Am J Cardiol 1974;34:627.

13. Cohen PR, Feigenbaum EA. The handbook of artificial intelligence. vol III. Los Altos, Calif.: William Kaufmann, Inc, 1981: $260-86$. 